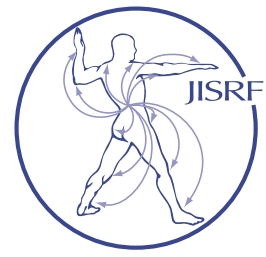

\title{
Femoral Head-Trunnion Dissociation in Metal-on-Polyethylene Total Hip Arthroplasty - A Unique Case Report
}

Patel $N^{1}$, Guild $G^{1}$, Erens $G^{1}$

\begin{abstract}
Background: Gross trunnion failure (GTF) after total hip arthroplasty is a rare complication and has only been reported in a few case series. Some of the associated risk factors have been described in the literature and include larger femoral heads, greater offset, and increased BMI. Despite this, the mechanism behind GTF is poorly understood and early diagnosis and treatment continues to be challenging.
\end{abstract}

Case Presentation: We present the case of complete femoral head and trunnion dissociation in a 63 year-old female nine years after total hip arthroplasty. Unique to this case is the lack of classic patient and implant risk factors for GTF along with the acute onset nearly nine years after implantation.

Discussion: This case presentation highlights the fact that the contributing factors and mechanism behind GTF continue to be poorly understood. There is a need for future research to help better understand this phenomenon and to help potentially identify those at risk for GTF.

\section{Background}

The use of femoral head modularity with a trunnion and bore has been the gold standard in hip arthroplasty due to ease of use, leg length and offset adjustability, and improved exposure in revision settings. Despite frequent use of modular components, corrosion at the head-neck junc- tion or trunnionosis has only recently received increased attention [1]. Severe trunnion corrosion can lead to mechanical deformation of the trunnion resulting in gross trunnion failure (GTF) [2]. The specific implant and patient factors that contribute to trunnionosis and GTF continue to be poorly understood. Several implant specific characteristics have been reported including titanium-alloy modulus, trunnion volume and contact area, taper-angle mismatch, varus-neck angle, high-offset, larger head, and dissimilar metals []]. Potential patient specific factors include high activity level, obesity, and male gender. [3] Incomplete trunnion cleaning at time of surgery and inappropriate impaction force of the head-neck have also been described [4]. We describe a case of GTF in a patient without the classically reported risk factors.

\section{Case Presentation}

At initial presentation in 2007, the patient was a 55 year old female with BMI of 22.8 and past medical history consisting of lumbar radiculopathy. She reported a multiple year history of progressive left sided hip and groin pain and was found to have end stage left hip osteoarthritis on radiographs (Figure 1). After failure of conservative measures, the patient underwent elective left total hip arthroplasty in

Keywords: hip arthroplasty, trunnion failure Level of Evidence: AAOS Therapeutic Level IV Educational Value \& Significance: JISRF Level B 

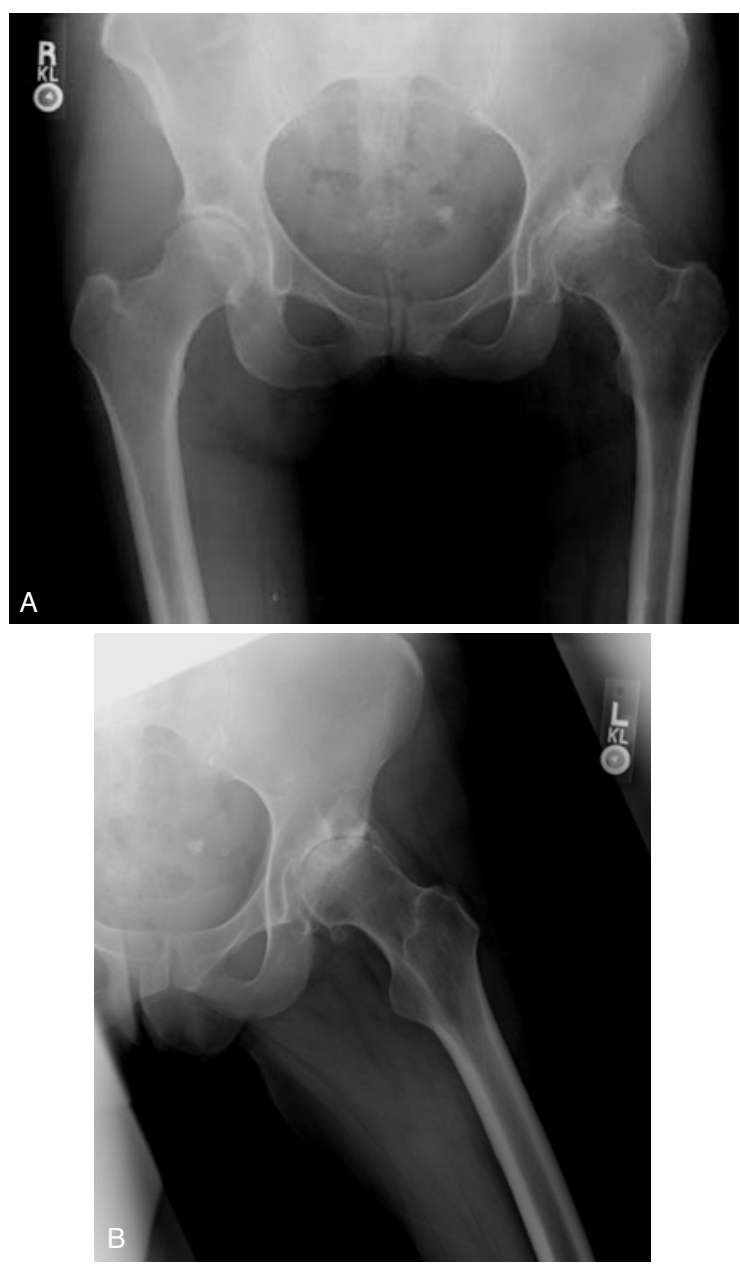

Figure 1. Preoperative AP pelvis (A) and left hip frog leg lateral (B) radiographs from May 2007 demonstrating advanced osteoarthritis of the left hip.

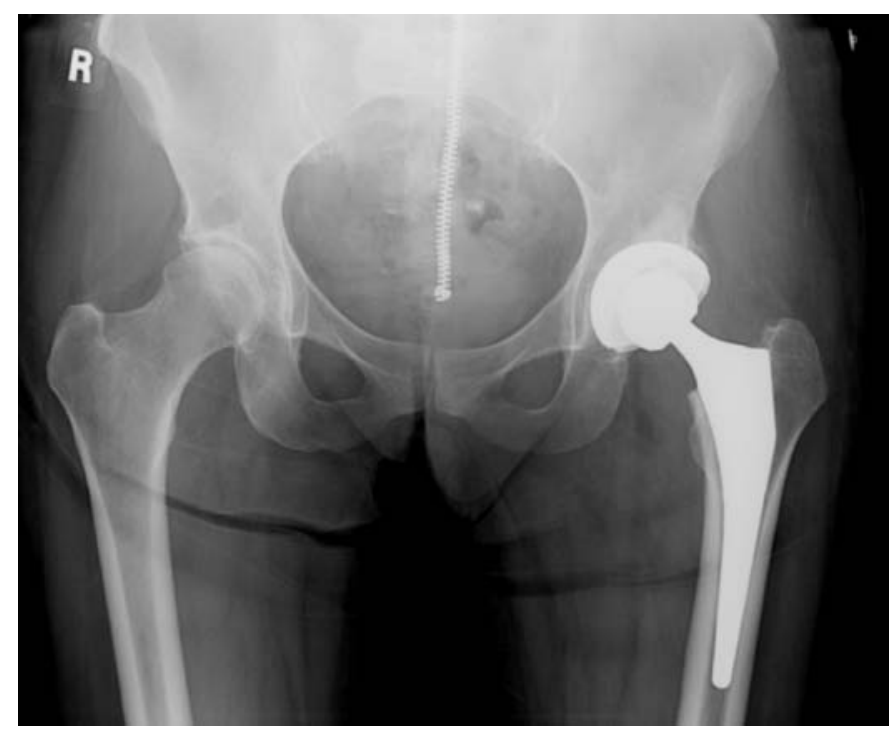

Figure 2. Initial post-operative AP pelvis radiograph from October 2007 after left total hip arthroplasty. Implants include Accolade I size 2.5 femoral component with $127^{\circ}$ neck angle, Trident $52 \mathrm{~mm} \mathrm{E}$ acetabular shell, $32 \mathrm{~mm}+4 \mathrm{CoCr}$ head ball, and X3 32mm, 0 degree polyethylene liner.
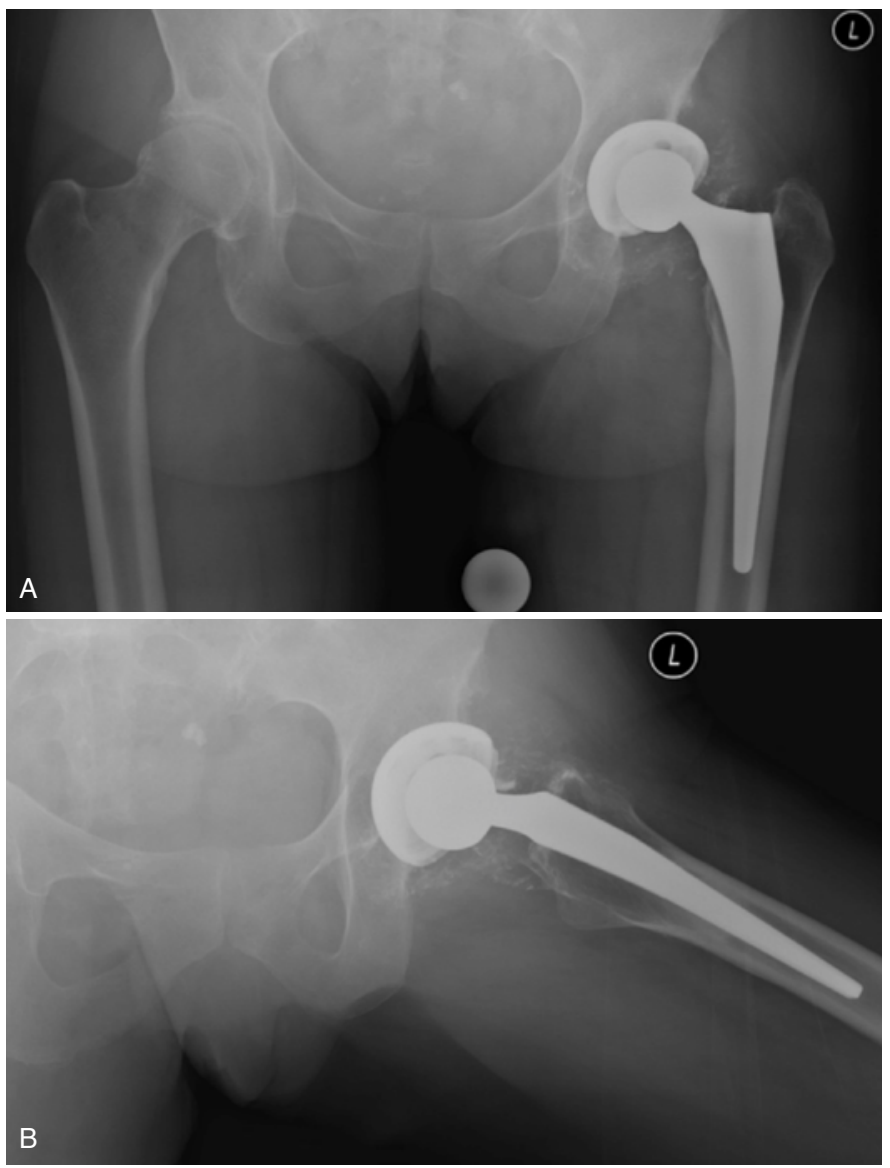

Figure 3. AP pelvis (A) and left hip frog leg lateral $(B)$ radiographs taken in July 2016, almost 9 years after initial implantation. Best visualized on the AP view, there is now notable change in angulation at the head ball trunnion interface from prior imaging with the head ball now residing in a more varus conformation. Additionally, metallic debris can be visualized outlining the left hip capsule.

October 2007 and tolerated the procedure well. Her hip implants consisted of an Accolade I TMZF size 2.5 femoral component with $127^{\circ}$ neck angle, Trident $52 \mathrm{~mm}$ E acetabular shell, $32 \mathrm{~mm}+4$ cobalt-chrome $(\mathrm{CoCr})$ head ball, and an X3 32mm, 0 degree crosslinked polyethylene liner (Stryker Mahwah, NJ) (Figure 2). She had an unremarkable post-operative course. The patient returned to clinic in 2016, almost nine years after her initial surgery, now 63 years old and with a BMI of 23.5. Her complaints included 3 weeks of painless clunking and left hip mechanical symptoms. Imaging revealed significant metallic debris about the hip joint along with interval alignment change at the head-trunnion interface with the head ball now residing in a more varus angulation (Figure 3). Based on these findings suggesting trunnion compromise, surgical intervention was felt warranted and revision hip surgery was scheduled. Several days after evaluation, the patient presented to the emergency room with acute onset of significantly worsened left hip pain and found to have complete dissociation of the femoral head and trunnion (Figure 4). 


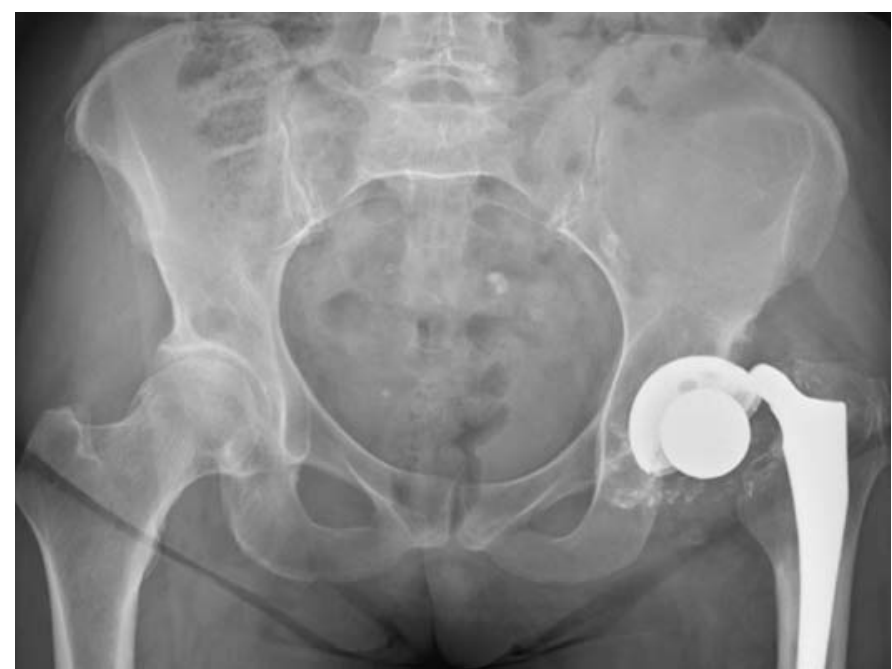

Figure 4. AP pelvis radiograph from August 2016 demonstrating complete femoral head ball and trunnion dissociation. Significant wear can be noted around the trunnion with again visualized metallic debris outlining the left hip capsule.
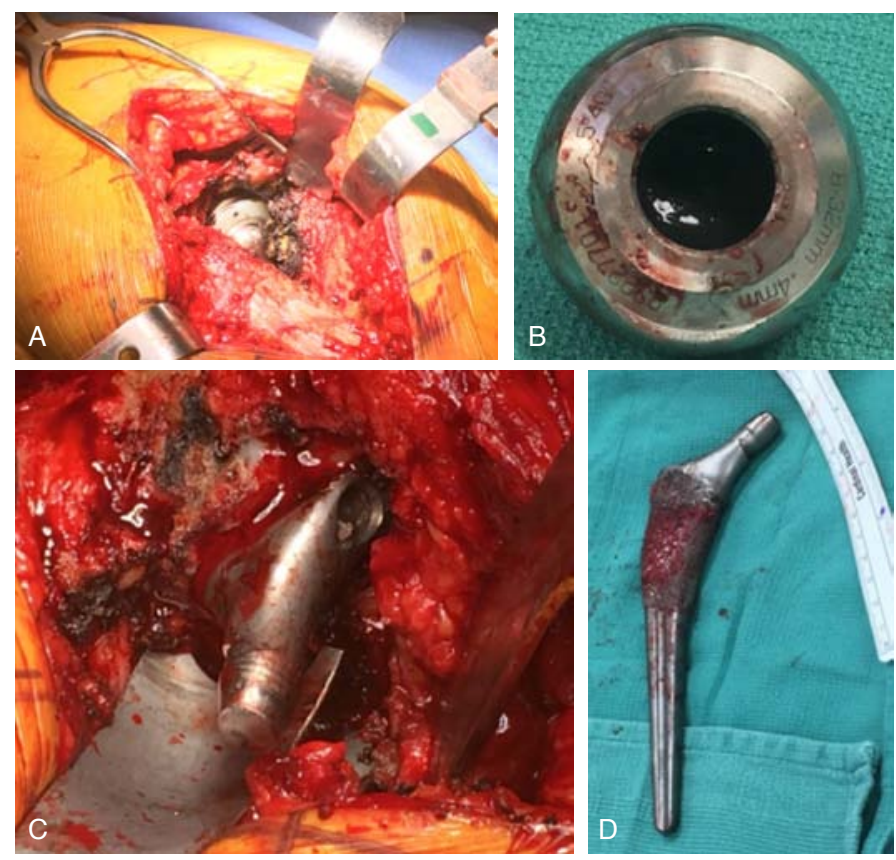

Figure 5. Intra-operative images from the revision left hip surgery in August 2016 (A) Posterior surgical approach to the left hip with obvious metallosis visualized in the soft tissues surrounding the implants. (B) $32 \mathrm{~mm}$ with $+4 \mathrm{~mm}$ offset $\mathrm{CoCr}$ head ball with evidence of corrosion and metallic debris inside. (C) In vivo and (D) explanted Accolade I size 2.5 femoral stem with significant wear noted about the trunnion.

The patient was admitted and underwent revision left hip arthroplasty without complication. Intraoperative images from her revision surgery are shown in Figure 5. She was found to have extensive metallosis in the soft tissues surrounding the left hip along with advanced corrosion and loss of material at the femoral trunnion. Her femoral component was replaced with a long stem modular implant fitted with a $36 \mathrm{~mm}$ ceramic head ball and her polyethylene

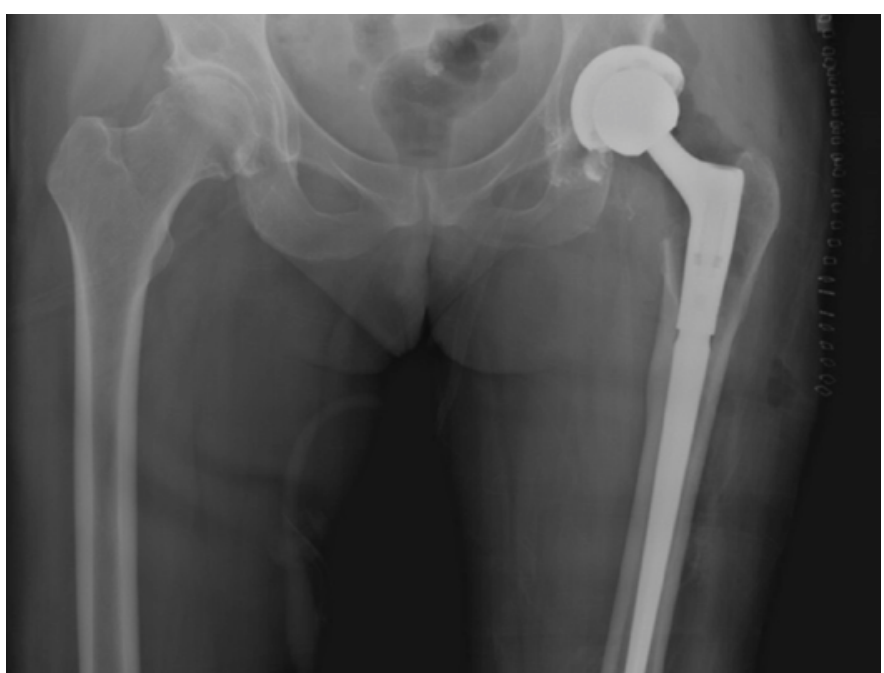

Figure 6. Post-operative AP pelvis radiograph after revision left total hip arthroplasty in August 2016. The patient's original femoral implant was replaced with a long stem modular component fitted with a 36mm ceramic head ball. The acetabular shell was left in place and fitted with a new 0 degree polyethylene liner.

liner was exchanged (Figure 6). Plasma chromium level was $10.0 \mu \mathrm{g} / \mathrm{L}$ (Ref range $0.1-2.1$ ) and serum cobalt level was $20.9 \mu \mathrm{g} / \mathrm{L}$ (ref range $0.0-0.9$ ). Since the revision surgery, the patient has done well with minimal hip pain and good mobility.

\section{Discussion}

Despite an increasing body of literature on trunnnionosis, there has been little reported on the unique mechanism of GTF. The few previous small case series have attempted to describe patient, implant, and surgeon factors that may contribute. In a series of five GTF's with the Accolade I TMZF stem (Stryker, Mahwah, NJ), Matsen et al. noted that male sex, BMI $>30$, head size $36 \mathrm{~mm}$ or greater, lateral offset $\left(127^{\circ}\right)$ neck angle, and increased head offset were commonly associated factors []․

In a case series involving multiple manufacturers, Banjeree et al. discuss the lack of data to implicate one single taper or neck geometry [2] . They too note the association with male gender and increased femoral offset. They surmised that the increased offset in the head may lead to stress and micro-motion in the taper with eventual fatigue and gross failure.

Confounding the issue have been reports of abnormal reactions to Beta titanium (TMZF) and concerns over its decreased modulus of elasticity being able to withstand cyclic loading. In a paper by Kirin et al., the authors postulate that the impaction of the femoral head or cyclic loading removes the oxide passivation layer creating crevices in the taper junction [6]. This allows for fluid ingress within the 
taper with depletion of oxygen and ionic changes ultimately leading to the formation of hydrochloric acid and resulting corrosion. It is possible that the decreased flexural rigidity of the TMZF trunnion contributes to crevice corrosion, as trunnions with higher modulus of elasticity have been shown to have less crevice corrosion [7]. Further confounders exist as there are studies that both support and refute the concept of taper size and geometry being associated with corrosion $[\underline{8}, \underline{2}]$. In a retrieval analysis, Cook et al. suggest that a combination of taper and trunnion angle mismatch, the use of a proximal contacting taper, and coupling of dissimilar metal alloys leads to taper damage and material loss [10].

Subsequent to the above mentioned reports, Styker Orthopaedics (Mahwah, NJ) issued a class II recall of 42,519 Co-Cr V40 LFIT head balls on August 29, 2016. Stryker received several complaints describing incidence of harm secondary to taper lock failure for specific lots of LFIT Anatomic CoCr V40 Femoral Heads thought to be a result of improper manufacturing tolerances. The patient in this case did not have a recalled implant.

What makes GTF evening more challenging to identify is the substantial time typically seen from implantation to failure. Walker et al. noted time to gross failure ranging from 4.4 to 7.6 years in a case series consisting of four patients [11]. Similarly, the time to trunnion failure observed in the presented case was nearly nine years after initial implantation.

This patient's demographics and implant characteristics are not typical of what has been reported in the literature with GTF and underscores the fact that the mechanism of femoral head-neck dissociation is still poorly understood. The patient in this case is female, with a BMI of 23.5 and an implantation time of nine years as compared to most other reports of male patients with BMI $>30$. This demonstrates that gender and BMI may be patient specific risk factors, but are not predictive of corrosion or failure. From an implant standpoint, the patient had a $32 \mathrm{~mm}$ femoral head, not the typical $36 \mathrm{~mm}$ or greater femoral head sizes suggested by previous authors as risk factors. The neck angle in this case is $127^{\circ}$ (varus neck) with an increased offset head (+4) on a Beta titanium stem with greater flexibility. These circumstances highlight that the cause of GTF may be multifactorial, but micromotion at the head-neck may be the common denominator. It remains difficult to suggest which patients should be monitored with X-rays, serum ion levels, and cross sectional imaging across all manufacturers. However, it is reasonable to identify patients who received recalled implants and provide surveillance with potential to intervene before GTF occurs. The typical lack of symptoms prior to GTF makes early diag- nosis and treatment challenging, and a high index of suspicion is required for early intervention. Future research is clearly needed to better understand this phenomenon and to help further identify those who are at risk for corrosion and GTF.

\section{References:}

1. Cooper HJ, Della Valle CJ, Berger RA, Tetreault M, Paprosky WG, Sporer SM,Jacobs JJ. Corrosion at the head-neck taper as a cause for adverse local tissue reactions after total hip arthroplasty. J Bone Joint Surg Am. 2012 Sep 19;94(18): $1655-61$.

2. Banerjee S, Cherian JJ, Bono JV, Kurtz SM, Geesink R, Meneghini RM, Delanois RE, Mont MA. Gross trunnion failure after primary total hip arthroplasty. J Arthroplasty. 2015 Apr;30(4):641-8. Epub 2014 Nov 26.

3. Jacobs JJ, Cooper HJ, Urban RM, Wixson RL, Della Valle CJ. What do we know about taper corrosion in total hip arthroplasty? J Arthroplasty. 2014 Apr;29(4): 668-9. Epub 2014 Feb 18

4. Gilbert JL, Mehta M, Pinder B. Fretting crevice corrosion of stainless steel stem$\mathrm{CoCr}$ femoral head connections: comparisons of materials, initial moisture, and offset length. J Biomed Mater Res B Appl Biomater. 2009 Jan; 88(1):162-73.

5. Matsen Ko L, Chen AF, Deirmengian GK, Hozack WJ, Sharkey PF. Catastrophic Femoral Head-Stem Trunnion Dissociation Secondary to Corrosion. J Bone Joint Surg Am. 2016 Aug 17;98(16):1400-4.

6. Kiran M, Boscainos PJ. Adverse reactions to metal debris in metal-on-polyethylene total hip arthroplasty using a titanium-molybdenum-zirconium-iron alloy stem. J Arthroplasty. 2015 Feb;30(2):277-81. Epub 2014 Oct 30.

7. Goldberg JR, Gilbert JL, Jacobs JJ, et al. A multicenter retrieval study of the taper interface of modular hip prostheses. Clin Orthop Relat Res 2002;401:149.

8. Panagiotidou A, Meswania J, Hua J, Muirhead-Allwood S, Hart A, Blunn G. Enhanced wear and corrosion in modular tapers in total hip replacement is associated with the contact area and surface topography. J Orthop Res. 2013 Dec;31(12):2032-9. Epub 2013 Aug 21

9. Higgs GB, MacDonald DW, Gilbert JL, Rimnac CM, Kurtz SM. Does Taper Size Have an Effect on Taper Damage in Retrieved Metal-on-Polyethylene Total Hip Devices? J Arthroplasty. 2016 Sep;(3):277-81. Epub 2016 Jul 6.

10. Cook, R.B., et al., Pseudotumour formation due to tribocorrosion at the taper interface of large diameter metal on polymer modular total hip replacements. J Arthroplasty, 2013. 28(8): p. 1430-6.

11. Walker, P., Campbell, D., Della Torre, P., Brazil, D., McTighe, T. Trunnion Corrosion and Early Failure in Monolithic Metal-on-Polyethylene TMZF Femoral Components: A Case Series. Reconstructive Review. Volume 6 (Number 3): September 2016

\section{SUBMISSION HISTORY}

Submitted September 9, 2017

Reviewed October 1, 2017

Revised December 6, 2017

Accepted December 7, 2017

Published December 29, 2017

AUTHOR AFFILIATIONS

1 Nick N. Patel, MD; George N. Guild III, MD; Greg A. Erens, MD Emory University School of Medicine, Department of Orthopaedic Surgery 59 Executive Park Dr NE, Atlanta, GA 30329

(Direct inquires to Nick N. Patel, nnpatel6@gmail.com)

AUTHOR DISCLOSURES

The authors declare there are no disclosures regarding the publication of this paper.

\section{COPYRIGHT \& OPEN ACCESS}

(C) 2017 Patel, Guild, Erens. All rights reserved. Authors retain copyright and grant the journal right of first publication with the work. Reconstructive Review is an open access publication and follows the Creative Commons Attribution-NonCommercial CC BY-NC. This license allows anyone to download works, build upon the material, and share them with others for non-commercial purposes as long as they credit the senior author, Reconstructive Review, and the Joint Implant Surgery \& Research Foundation (JISRF). An example credit would be: "Courtesy of (senior author's name), Reconstructive Review, JISRF, Chagrin Falls, Ohio". 